\title{
Internacionalización de las pymes: análisis de recursos y capacidades internas mediante lógica difusa
}

\author{
Internationalization of SMEs: analysis of resources and internal \\ capabilities using fuzzy logic
}

Jorge Aníbal Restrepo Morales y Juan Gabriel Vanegas López*

Tecnológico de Antioquia Institución Universitaria, Facultad de Ciencias Administrativas y Económicas, Colombia

Recibido el 17 de septiembre de 2014; aceptado el 5 de diciembre de 2014

Disponible en Internet el 20 de agosto de 2015

\section{Resumen}

Las pymes del sector textil enfrentan grandes desafíos de internacionalización derivados de la dinámica de la globalización y de la firma de tratados de libre comercio en la última década. Para ello se requiere analizar su capacidad de internacionalización en términos de recursos, habilidades y capacidades para desarrollar estrategias que permitan participar de la nueva dinámica en una forma apropiada. Se propone un sistema de inferencia difuso para modelar estos factores que determinan el éxito en un proceso de internacionalización. Se utilizan variables lingüísticas, extraídas de empresarios, expertos, consultores e investigadores de la materia y, por medio del juicio de expertos, se definen los factores internos que explican la capacidad de internacionalización. El modelo propuesto se aplica por medio de un estudio de caso del clúster textilconfección en Medellín (Colombia). Se obtiene en la ejecución del modelo un índice global particular de 26.7 para las capacidades internas para iniciar un proceso de internacionalización. El resultado obtenido confirma la hipótesis de que las capacidades y recursos de que dispone actualmente el sector analizado no son suficientes para una inserción exitosa en el mercado internacional y, lo más importante, especifica los factores y variables en los que intervenir para mejorar la capacidad interna de internacionalización.

Derechos Reservados (C 2015 Universidad Nacional Autónoma de México, Facultad de Contaduría y Administración. Este es un artículo de acceso abierto distribuido bajo los términos de la Licencia Creative Commons CC BY-NC-ND 4.0.

Palabras clave: Conjuntos difusos; Capacidad exportadora; Estrategia de negocios; Sector textil; Pymes Códigos JEL: C65; D22; F14; M11; O54

\footnotetext{
* Autor para correspondencia.

Correo electrónico: jg.tecnologico@gmail.com (J.G. Vanegas López).

La revisión por pares es responsabilidad de la Universidad Nacional Autónoma de México.
} 


\begin{abstract}
SMEs in the textile and apparel sector face major internationalization challenges arising from the dynamics of globalization and the signature of free trade agreements during the last decade. It is then necessary to analyze the export capability of these enterprises in terms of the resources, skills, and abilities needed to participate successfully in the international scene. A fuzzy inference system is proposed to model the resources, skills, and capabilities that determine export success. Linguistic variables, collected from entrepreneurs, experts, consultants, and researchers in the field, were used to define the internal factors that explain export capabilities. The proposed model is validated by using the textile and clothing cluster in Medellin, Colombia, as a case study. The model reports a particular global index of 26.7 for export capabilities. On the one hand, the result confirms the hypothesis that the capabilities and resources currently available to the sector are not sufficient for a successful integration into the international market, and most importantly, on the other hand, it specifies which factors and variables are important to improve the export capability of the sector.

All Rights Reserved (c) 2015 Universidad Nacional Autónoma de México, Facultad de Contaduría y Administración. This is an open access item distributed under the Creative Commons CC License BY-NC-ND 4.0.

Keywords: Fuzzy set methods; Export capabilities; Business strategy; Textile and apparel sector; Small and medium-sized enterprises

JEL classification: C65; D22; F14; M11; O54
\end{abstract}

\title{
Introducción
}

Para el caso colombiano, se plantea que el modelo de internacionalización debe considerar el fortalecimiento del entorno empresarial para abordar un proceso de globalización gradual y exigente, tomando el tema de la exportación como el primer paso para posicionarse en el escenario mundial. Sin ser la única base, el proceso debe surgir de un contexto histórico local y fortalecer el desarrollo interno de la empresa para enfrentar los mercados internacionales y sus retos con la capacidad que esto demanda. Esta realidad requiere de herramientas prácticas para el desarrollo estratégico internacional de las pymes en Colombia, que permitan afrontar los retos que implica cualquier proceso de integración al que se vincule el país.

A nivel mundial, Colombia es ampliamente reconocida por sus ventajas significativas como productor de textiles y prendas de vestir. Esta industria representa cerca del $8 \%$ del producto interno bruto (PIB) industrial y el 3\% del PIB nacional. Además, contribuye con el 13\% de las exportaciones de manufacturas y es responsable del $24 \%$ del empleo industrial. La industria textil y del vestido está compuesta por alrededor de 10,000 fábricas localizadas en 7 ciudades. Medellín absorbe alrededor del $40 \%$ de las empresas del sistema, lo que la ubica en la cima de la industria textil en Colombia, generando más del 6\% del PIB industrial (Inexmoda, 2011).

Comparativamente, a nivel internacional, la industria textil colombiana evidencia una baja penetración en la participación de las exportaciones de este sector, lo cual pone de manifiesto la necesidad de repensar la estrategia de negocio bajo la luz de la evidencia empírica reflejada por la debilidad o fortaleza de los factores críticos del éxito exportador. En este orden de ideas, este artículo propone un modelo para crear un índice global de evaluación de la capacidad de exportación de las empresas, recurriendo a variables cualitativas de naturaleza linguiística. Para ello se propone un sistema de inferencia difuso (FIS), como un enfoque de análisis flexible, que 
permite realizar una evaluación integral de los recursos y capacidades internas para iniciar un proceso de internacionalización de las empresas de una manera sistemática.

Las relaciones entre variables explicativas de dicha capacidad se definen usando la técnica de criterio de expertos y se consideran los factores internos más relevantes, a saber: marketing, logística, gestión de planificación, planificación de recursos humanos y financieros, entre otros, para conseguir un mayor grado de objetividad en la medición. Cada factor crítico de éxito se compone de un conjunto de entre 10 y 12 variables lingüísticas específicas cuyas relaciones se definen mediante la valoración de empresarios, académicos, usuarios y expertos en comercio internacional. El sistema de modelación FIS permite correlacionar las variables linguísticas y ajustar los datos para generar un índice numérico global que represente y permita evaluar las capacidades de internacionalización de pequeñas y medianas empresas (pymes) en la industria textil-confección de Medellín (Colombia).

Este artículo se compone de 5 secciones incluida esta introducción. En la sección 2 se presenta una revisión teórica sobre la internacionalización de las empresas y la importancia del proceso exportador para su desarrollo. Igualmente, muestra los antecedentes teóricos y aplicaciones de los modelos de la lógica difusa. La sección 3 aborda la teoría del sistema de inferencia difusa y la sección 4 presenta los resultados del estudio de caso para la industria textil-confecciones donde se aplicó este método; por último, se presentan las conclusiones, recomendaciones y limitaciones.

\section{Revisión de la literatura}

\section{Estudios empíricos y perspectivas teóricas en torno a los recursos y la internacionalización de las empresas}

La internacionalización de la empresa, como fenómeno económico, ha despertado el interés investigativo desde diversas aristas. Santiso (2007) estudia las empresas de la economía española, y muestra como en la década de los noventa, se convirtieron en jugadores globales tomando a América Latina como trampolín internacional para la conversión de las empresas españolas. En Argentina, se analiza el proceso de internacionalización de un grupo de empresas industriales a partir de la instalación de plantas en el exterior; presentan como hipótesis que el proceso, más que un fenómeno puntual y aleatorio, es el resultado de un complejo de causas relacionadas con las características y dinámica de la estructura productiva interna (Bisang, Fuchs, y Kosacoff, 1992,).

Otros estudios abordados indican como denominador común que la internacionalización significa el conjunto de operaciones para establecer vínculos, con cierto grado de estabilidad, entre la empresa y los mercados internacionales, por medio de un proceso incremental de implicación y proyección internacional (Root, 1994; Rialp, 1999). Por su parte, Cruz (2010) realiza una investigación empírica en 100 empresas de 3 países: Argentina, Bolivia y Panamá, seleccionados en función de la facilidad de acceso a las empresas. En él concluye que la gran mayoría de las empresas $(65 \%)$ indica que el acceso a la internacionalización ha sido gradual; mientras que una minoría (11\%) indica que ha sido a gran velocidad; en tanto que una fracción importante de las entrevistadas (24\%) manifestó su condición de empresas «nacidas globales».

En cuanto al enfoque de recursos, Escandón y Hurtado, 2013 señalan que la decisión de incursionar en un mercado foráneo surge del análisis de la estrategia global considerada para competir internacionalmente, la cual depende de la ventaja competitiva en recursos y capacidades del sector, del país de origen o de alianzas estratégicas. Los recursos y la propiedad de ventajas específicas son factores importantes para el proceso de internacionalización de una firma (Gaur, Kumar y Singh, 2014). Es menester enfatizar que una ventaja sostenible demanda no solo la 
propiedad de activos y conocimientos difíciles de replicar, sino que también requiere una exclusiva batería de capacidades dinámicas, las cuales pueden ser adquiridas y continuamente creadas, extendidas, protegidas y mantenidas como una base de activos única (Teece, 2007). En el trabajo de Lin y Wu (2014), encuentran que las capacidades dinámicas de aprendizajes media de forma más eficaz la consolidación de los recursos más valiosos, raros, inimitables y no sustituibles con que cuenta una firma.

Para el caso colombiano, Castro (2007) plantea que el modelo de internacionalización debe considerar el fortalecimiento del entorno empresarial colombiano para abordar un proceso de globalización gradual y exigente. Argumenta que se ha tomado el tema de la exportación como el primer paso para la internacionalización, pero que no puede ser la única base: el proceso debe surgir de un contexto histórico local y debe fortalecer el desarrollo interno de la empresa para enfrentar los mercados internacionales y sus retos con la capacidad que esto demanda. Concluye que, a través de herramientas prácticas para el desarrollo estratégico internacional de las pymes en Colombia, se lograrán afrontar los retos que implica cualquier proceso de integración al que se vincule el país.

Ahora bien, desde los diferentes enfoques teóricos propuestos que intentan explicar el proceso de internacionalización de las empresas, se toman en consideración 3 perspectivas. En primera instancia, la perspectiva económica que considera la internacionalización de la empresa mediante el análisis de la globalización, las empresas multinacionales y sus operaciones y, más específicamente, por medio de las actividades de las empresas transnacionales, a la que pertenecen las teorías que detallan el proceso de internacionalización a través de una figura que se han enfocado en los costos y las ventajas económicas de la internacionalización (Rialp, 1999; Gómez Suárez, González, Lusa, y Osorio, 2006).

En segundo lugar, la teoría de recursos y capacidades (Wernerfelt, 1984) trata la internacionalización como un compromiso de aprendizaje incremental basado en la acumulación de conocimiento y la incorporación de recursos y habilidades para la orientación a los mercados externos; se presenta el caso particular del modelo Uppsala y sus derivaciones y adaptaciones (Johanson y Wiedersheim-Paul, 1975; Alonso, 1994; Rialp, 1999; Gutiérrez y Heras, 2000).

En tercer lugar, surge la teoría de redes, las comunidades clústeres y el proceso de internacionalización (Thomas y Araujo, 1985; Thorelli y Cavusgil, 1990). Por su importancia se deben considerar las nuevas concepciones que asimilan el proceso de internacionalización como un desarrollo lógico de las redes interorganizativas y sociales de las empresas, categoría que ubica el caso particular del concepto de clúster (Johanson y Mattson, 1988; Blankenburg Holm, 1995).

En la década de los 80 del siglo pasado, se acentúa la visión estratégica de que los factores internos de la empresa facilitan o entorpecen el camino hacia la internacionalización. La teoría de recursos y capacidades expone las circunstancias que explican como empresas que operan en entornos competitivos similares y con factores de éxito comunes para el sector económico generan en promedio rentabilidades superiores y diferenciadas (Huerta, Navas y Almodóvar, 2004).

En este orden de ideas, la gestión de las empresas en los mercados internacionales depende de sus factores internos, en especial de las capacidades y recursos disponibles, partiendo de la premisa de que los beneficios obtenidos por una empresa están en función de los factores externos (características competitivas del entorno) y los factores internos (la integración de los recursos de que dispone). Estos últimos son quizá el mayor reto de esta investigación, porque se requiere proponer una batería adecuada de capacidades y definir su importancia y relevancia estratégica para la construcción de ventajas competitivas (Wernerfelt, 1984).

En general, la teoría de soporte considera las capacidades como las habilidades y procedimientos organizacionales, tales como: los procesos, activos, sistemas de información y el conocimiento 
que habilitan a una empresa para formular estrategias apropiadas para competir internacionalmente (Barney, 1991; Claver y Quer, 2001). En este contexto, uno de los retos de la dirigencia empresarial radicará en identificar, desarrollar, proteger y desplegar recursos y capacidades de una forma tal que la empresa obtenga una ventaja competitiva sostenible que le proporcione un retorno superior (Amit y Schoemaker, 2012; Tallman y Fladmoe-Lindquist, 2002).

En síntesis, siguiendo los planteamientos de Hamel y Prahalad (1994), la posición competitiva de una empresa será una función directamente proporcional a la forma como combina sus recursos y capacidades, sin dejar de lado que lo que se espera para una empresa va más allá del conjunto de capacidades que puede desarrollar, lo que significa que la estrategia de la firma adquiere una nueva dimensión que implica, por ejemplo, que la competencia de las firmas no se sitúe en el desarrollo de nuevos productos sino en la capacidad incorporada para desarrollar estos. Esto implica que la estrategia debe reconocer como la firma requiere desaprender mucho de su pasado antes de encontrar su futuro. Por lo tanto, la estrategia se preocupa menos de obtener un ajuste preciso entre las metas y los medios y más de crear metas flexibles que reten a los empleados; la estrategia es más que alinear los recursos escasos por medio de proyectos competitivos y encontrar cómo superar las restricciones de estos por medio de una indefinida y creativa búsqueda de un mejor uso de los recursos en promedio.

La evidencia parece confirmar que la generalidad de los procesos de internacionalización de las pymes son fenómenos esencialmente evolutivos en los cuales, a través del tiempo, se concentran habilidades y conocimientos que viabilizan la superación de barreras y permiten alcanzar niveles competitivos superiores; esa evolución está íntimamente ligada a la incorporación de conocimientos y a la sofisticación de la gestión. En ese orden de ideas, este trabajo presenta una hipótesis que se apropia de la teoría de recursos y capacidades como modelo de aclaración. Dicha teoría data de 1984 y esgrime como propósito analizar los recursos y capacidades para identificar el potencial de una firma para instaurar ventajas competitivas a través de identificar y valorar los recursos y habilidades disponibles, ya sea porque los posee o tiene posibilidades de alcanzarlos, se enfatiza la importancia de este análisis para explicar la evolución de sus resultados (Wernerfelt, 1984).

\section{Modelo de inferencia difuso}

La lógica difusa, al contrario de lo que su nombre indica, es un raciocinio alternativo a la lógica clásica que pretende calificar información imprecisa, es decir con cierto grado de vaguedad, haciendo énfasis en que las borrosas son las variables, que representan esta información, y no el modelo como tal (Zadeh, 1965; 2008). En el entorno cotidiano, mayormente empresarial, gran parte del conocimiento es disonante con la realidad, es decir, impreciso, vago, ambiguo, inexacto, incierto o probabilístico por naturaleza. El razonamiento -por la vía de la reflexión y el pensamiento de los seres humanos-, en un alto porcentaje, contiene información de este tipo, originada en la inexactitud intrínseca de los conceptos y del raciocinio desprendido de experiencias similares (Dourra y Siy, 2002; Darshan, Jagdev y Om Pal, 2012).

En la actualidad, la lógica difusa o borrosa atrae a un gran número de seguidores, debido a que utiliza expresiones que no son ni cabalmente ciertas ni plenamente falsas, es decir, es la lógica adaptable a conceptos que pueden tomar un valor cualquiera de veracidad en un conjunto de valores que fluctúan entre 2 extremos: la verdad absoluta y la falsedad total. Es importante clarificar que lo que es difuso, complejo o impreciso no es la lógica en sí, sino el objeto que pretende estudiar: expresa la falta de definición del concepto al que se aplica (Zadeh, 2008). La lógica difusa trata información imprecisa, el margen de contribución de una empresa es bajo o la 
volatilidad de la tasa de cambio es alta, en términos de conjuntos borrosos que se combinan en reglas para definir acciones: si proposición 1, entonces proposición 2. Ejemplo: si la volatilidad de la moneda es alta, entonces el margen de ganancia de las empresas exportadoras es bajo. Así las cosas, los sistemas de control difuso ajustan variables de entrada, definidas en términos de conjuntos difusos, por medio de grupos de reglas que originan uno o varios valores de salida.

Un sistema basado en lógica difusa, como consecuencia de la simplicidad de los cálculos exigidos (básicamente sumas y comparaciones), permite aprovechar el conocimiento de los expertos en un tema, como la base para alcanzar una optimización automática al formalizar el conocimiento ambiguo que se desprende del sentido común, de una forma operable (Martín del Brio y Sanz, 2002). Los modelos de lógica difusa son agudamente flexibles, tolerantes a la imprecisión de los datos y posibilitan manipular funciones no lineales de diversa complicación y, lo más importante, no dependen de supuestos estadísticos alrededor de las características de los datos y sus distribuciones de probabilidad. Ante la presencia de información imprecisa e insuficiente, el uso de instrumentos estadísticos ni garantiza, ni es suficiente para obtener resultados significativos. Una combinación de un sistema de lógica difusa con la experiencia o conocimiento desarrollada e incorporada en un grupo de expertos, en el proceso de la toma de decisiones, es una extraordinaria forma de conquistar resultados positivos (Kosko, 1994).

Ahora bien, la aplicación de los conjuntos difusos se ha difundido ampliamente para el diseño y respaldo de la toma de decisiones en múltiples áreas del conocimiento: evaluación de proyectos, evaluación de riesgos operativos, detección de fallas o fraudes, sistemas de diagnóstico en aplicaciones médicas, sistemas de diagnóstico en psicología y sociología, aplicaciones en mercadeo, controladores de equipos industriales, evaluación sociopolítica y control de calidad, entre otros (Medina, 2006). Mendoza (2009) desarrolla una aplicación, en el ámbito social, sobre la percepción de los actores de una empresa acerca de la autonomía y eficiencia de los trabajadores. Wi y Jung, 2014 proponen una aplicación para relacionamientos sociales basada en el conocimiento para distinto tipo de organizaciones. Rush, Bessant, y Hobday, 2007 proponen el desarrollo de una evaluación y auditoría de capacidades en materia de tecnología. Aguirre (2010) expone una metodología para medir y evaluar las capacidades tecnológicas de innovación y su impacto en el desempeño de empresas de ingreso tardío al mercado; capacidades que también se han modelado en el sistema universitario (Serrano, 2010; Veltri, Mastroleo, y Schaffhauser-Linzatti, 2014).

En las decisiones empresariales en el área financiera, de producción y toma de decisiones se encuentra como Dourra y Siy (2002) que parten de los indicadores financieros y generan nuevas variables que son parametrizadas mediante conjuntos difusos para aplicar en decisiones e inversión. Medina y Paniagua (2008) desarrollan un trabajo en una cooperativa de servicios financieros con un FIS para evaluar la solvencia de los asociados solicitantes de crédito. Torabi y Hassini (2008) se orientaron a la creación de un modelo para integrar los distintos niveles de la cadena de suministros, considerando múltiples proveedores, un fabricante y diversos centros de distribución, teniendo en cuenta que la demanda del mercado, el costo por periodo y los niveles de capacidad son variables imprecisas. Björk (2009) plantea un modelo para la determinación de la cantidad económica de pedido utilizando pedidos diferidos; en él la demanda y los leed times son parámetros difusos. El trabajo desarrollado por Wang y Liang, 2004 presenta un novedoso e interactivo modelo de programación lineal probabilística para solucionar un problema de planeación agregada con incertidumbre en la demanda prevista, gastos de funcionamiento y capacidad. También existe una aplicación para determinación y proyección de la demanda, combinando las proyecciones del mercado y la entrega (Wanga y Chang, 2010). Sánchez, Herrera, y Herrera-Viedma (2009) corroboran la importancia de una planificación de recursos empresariales en las organizaciones para integrar de una forma adecuada la información. Vergara y Gaviria (2009) demostraron la 
aplicabilidad que posee la lógica difusa para resolver problemas de planeación de la producción. Marchi, Vignola, Facchinetti, y Mastroleo (2014) modelan la selección de mercados internacionales por parte de pequeñas empresas. Arango, Serna, y Pérez, 2012 desarrollan una aplicación en la gestión de indicadores empresariales para la toma de decisiones que propone un sistema de medición y análisis basado en el Balance Scorecard, incorporando técnicas de lógica difusa para disminuir la incidencia de la incertidumbre en dichos procesos. Cejas-Montero (2011) utiliza la lógica difusa como un modelo lógico para la modelación simultánea de los procesos deductivos y de toma de decisiones empresariales.

\section{Método}

\section{Hipótesis}

El proceso normal de transformación de una empresa local en internacional es gradual, en el que el nivel de compromiso de la empresa crece paralelamente con su conocimiento del mercado externo y las variables inherentes a su propio proceso. Como resultado, la expansión internacional de la compañía consiste en un conjunto de pasos secuenciales, en los que inicialmente se instalan fuera de sus fronteras aquellos procesos más cercanos a las actividades de cadena de valor del cliente, como es el caso de las exportaciones, enmarcado como una actividad de comercio exterior para, a partir de allí, iniciar un proceso de internacionalización asumiendo mayores retos y compromisos. Como se ha establecido que la conducta de las empresas en los mercados internacionales depende de los factores internos a ella, en el caso particular de las pymes del sector textil/confección de la ciudad de Medellín, se afirma que las capacidades y recursos de que dispone actualmente no son suficientes para una inserción exitosa en el mercado internacional. Por tanto, se requiere adoptar estrategias para mejorar aspectos internos en los que se presentan debilidades, potenciar aquellos en los que existen fortalezas y facilitar la incorporación de las ventajas competitivas requeridas para el posicionamiento internacional.

\section{Diseño de la investigación}

Los objetivos del estudio se limitaron a la ciudad de Medellín como el principal productor de textiles, así como un centro internacional de la moda en Colombia. Esta es una investigación de corte analítico, con una orientación de corte mixto -de tipo cualitativo y cuantitativo-, en la cual se pretende, a partir de un conjunto de variables endógenas, elegidas mediante el método Delphi por un grupo de expertos del sector textil/confección, medir las capacidades y recursos que una firma necesitaría para abordar el proceso de internacionalización.

Para definir el método, se recurrió a un estudio elaborado por Vázquez y Vázquez (2007), en el que analizaron los trabajos empíricos y enfoques publicados entre 1999 y 2004, y que presentan como foco de atención de forma específica la internacionalización de las pymes. En la pesquisa abordaron 29 trabajos y el criterio de selección fue la rigurosidad científica. Concluyen que las técnicas y metodologías más recurrentes en los estudios señalados se limitan a los cuestionarios y las encuestas en profundidad para los estudios de caso, dentro de los cuales el $90 \%$ utilizaron esta técnica. Frente a los estudios empíricos, concluyen que las técnicas más empleadas por los investigadores fueron los análisis de regresión múltiple, análisis de regresión logarítmica, análisis de varianza, análisis multivariantes o combinaciones de algunos de estos.

En vista de la heterogeneidad de los estudios, se utiliza un amplio portafolio de variables que sería muy complejo de modelar con técnicas tradicionales. Por esta razón, se elige un modelo 
multicriterio basado en lógica difusa, debido a que es una técnica que se ha empleado con bastante éxito en la industria, y cada vez se está usando en multitud de campos, fundamentalmente porque no requiere de valores exactos, tolera valoraciones subjetivas de tipo lingüístico para los parámetros del problema y las agrega convenientemente a modelos matemáticos complejos (Medina, 2006). Entre las características diferenciadoras de la lógica difusa podemos mencionar: soporta datos imprecisos, es conceptualmente fácil de entender, flexible, basada en el lenguaje humano y en la experiencia de expertos conocedores del problema en cuestión (Zadeh, 1965; Bellman y Zadeh, 1970). Así, se pueden modelar funciones no lineales de alguna complejidad y combina en forma unificada expresiones lingüísticas con datos numéricos, como es el caso de la relación entre las 157 variables elegidas para explicar la capacidad exportadora (Ver Anexo de variables explicativas), que exige utilizar modelos no tradicionales que asienten una aproximación a variables de corte cuantitativo, transformando la solución del problema en una situación menos imprecisa.

Un sistema de lógica difusa se caracteriza porque posibilita utilizar el conocimiento de expertos en un área, como base para la automatización de procesos al formalizar dicho conocimiento, ambiguo en algunos casos, en un algoritmo realizable compuesto de cálculos de sumas y comparaciones (Martín del Brio y Sanz, 2002). Es así como la metodología aplicada permite representar tanto la percepción de los empresarios de pymes como las propuestas desarrolladas en la literatura y el juicio de expertos en el tema, para evaluar los recursos y capacidades que las empresas requieren en su evolución hacia un adecuado desempeño en internacionalización. Para ello, se recurre a la modelación de variables lingüísticas con las que se genera un valor numérico que representa dicha capacidad y cada uno de los factores que lo componen. Al respecto, Kaleka (2002) determina que ninguno de los factores, de forma independiente, se configura como un factor crítico en el proceso, de hecho es el efecto generado por la sinergia de su combinación lo que importa más, lo que justifica aplicar un sistema de lógica difusa para generar un indicador integral que represente la sinergia de las variables consideradas. Es así como, de manera sistémica, se hace una valoración integral con el propósito de tamizar las relaciones entre las variables explicativolingüísticas de dicha capacidad y de alcanzar mayor objetividad en la medición ajustada a los sistemas de percepción y valoración de los empresarios.

Los factores claves del éxito de internacionalización se determinan mediante la técnica de construcción de diagramas de afinidad. Se diseñan, además, los conjuntos difusos, las reglas de decisión, la base de conocimiento y los mecanismos de validación y posterior intervención, a partir de un grupo de empresas seleccionadas, y de la aplicación de un cuestionario con variables lingüísticas, definidas por un panel de expertos y empresarios. Finalmente, se aplica la métrica para cuantificar y evaluar la capacidad de internacionalización, la cual se genera con el software Matlab.

Metodológicamente, el trabajo se dividió en 3 etapas:

1. Construir un diagrama de afinidad, tomando como guía las capacidades de innovación tecnológica, expuestas por Yam et al. (2004), Cheng et al. (2006) y la cadena de valor de Porter (1985).

2. Construcción de un modelo de Excel, focalizado en 18 factores y 157 variables lingüísticas calificadas por un grupo de expertos. La base informativa inicial fue definida, en primera instancia de la revisión bibliográfica de los factores críticos de éxito considerados por consultores y escritores de temas empresariales y, mediante una lluvia de ideas. En este proceso, se recabaron 12 factores, 25 subfactores y 250 variables; posteriormente, se invitó a un grupo compuesto por 10 representantes legales del clúster de confecciones, agremiados en la Asociación Colombiana de Pequeños Industriales (ACOPI), y por el método de scoring se calificó 


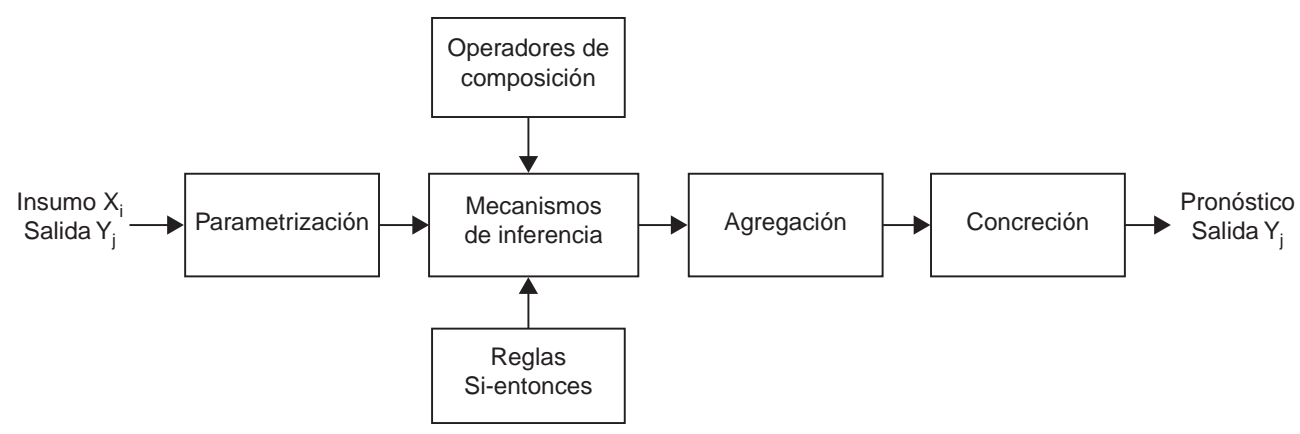

Figura 1. Pasos para construir un FIS.

Fuente: Jang et al. (1997).

la importancia de estos, hasta reducir la base informativa inicial a 8 factores, 18 subfactores y 157 variables. Luego, se conformó el grupo de expertos con los siguientes miembros: el coordinador regional de la Asociación Nacional de Empresarios de Colombia (ANDI) para el sector textil en el departamento de Antioquia; el director de ACOPI; 5 representantes de los empresarios de pymes del sector de confecciones y 3 consultores en planeación estratégica. Con base en estas percepciones, se definen funciones de pertenencia a conjuntos de fortalezas mayores, fortalezas menores, debilidades mayores y debilidades menores en un rango de [0-40].

3. Diseñar un sistema de inferencia difusa Mamdani (Mamdani, 1977), basado en obras de Medina (2006) y propuesto por Kosko (1994), Jang, Mizutani y Sun (1997) y Kasabov (1998).

\section{Recolección de información}

Los principales mecanismos para la recolección de información fueron encuestas, entrevistas semiestructuradas, observaciones dirigidas y actividades participativas. Para determinar las empresas de la muestra, se utilizó la base de empresas afiliadas a ACOPI y se seleccionaron 13 empresas para el estudio piloto mediante un muestreo por conveniencia. Se realizaron encuestas a los representantes legales de la empresa o, alternativamente, a quien ejecutara la función de planificación estratégica de negocios.

\section{Conjuntos difusos y sistemas de inferencia difusos}

Los FIS convierten variables de entrada (cuantitativas o cualitativas) en variables lingüísticas, a través de funciones de pertenencia o conjuntos difusos, los cuales son evaluados mediante un conjunto de reglas difusas del tipo condicional (if-then); luego estas variables adquieren valores concretos mediante un proceso conocido como defuzzyfication y de esta manera es posible analizar la información para la toma de decisiones (Bellman y Zadeh, 1970).

Los pasos para crear un FIS se proponen en la figura 1, se puede encontrar información de todo el desarrollo en Jang et al. (1997), Kosko (1994) y Medina (2010). A continuación, se enumeran las diferentes fases del diseño del FIS, adaptado de acuerdo con el proceso desarrollado de este trabajo:

1. Identificación del tipo de problema y el tipo de sistema difuso que mejor se ajusta a los datos. 
2. Definición de variables de entrada y salida, sus valores difusos y sus funciones de pertenencia (parametrización de variables de entrada y salida).

3. Definición de la base de conocimiento o reglas difusas.

4. Ajuste del sistema, validando los resultados a partir de la prueba de escritorio.

5. Obtención de salidas del sistema mediante la información de las variables de entrada, utilizando el FIS, el cual utiliza operadores de composición.

6. Traslado de la salida difusa del sistema a un valor nítido o concreto, mediante un sistema de defuzzificación.

\section{Limitaciones de los conjuntos difusos}

Rajendra y Priti, 2009 mencionan como limitaciones del sistema de lógica difusa lo siguiente:

1. Los sistemas de lógica difusa carecen de las habilidades de aprendizaje de las máquinas, así como de los patrones de reconocimiento de memoria (tipo red neural). Por esta razón, los sistemas híbridos (por ejemplo, los sistemas neurofuzzy) son cada vez más populares para aplicaciones específicas.

2. Definir exactamente las funciones de membresía y las reglas borrosas es una tarea difícil. En la mayoría de los casos es imposible predecir cuántas funciones de pertenencia se requieren, incluso después de amplias pruebas.

3. Confirmar y probar un sistema basado en el conocimiento difuso generalmente exige de pruebas exhaustivas y con alto requerimiento de software, que algunas veces es un asunto de costos.

4. La estabilidad del sistema es una preocupación importante para el control borroso.

\section{Implementación y resultados}

\section{Definición de la base de conocimiento para las reglas difusas}

En primera instancia, en las sesiones de trabajo con expertos en comercio internacional y con un proceso de intercambio de ideas, se definieron 18 subfactores (fig. 2) que actúan como proxy para explicar las habilidades y recursos que deben gestionar las pymes en su proceso de exportación y que resumen las 157 variables linguísticas definidas en la etapa 1 de la metodología. Luego, con la elaboración del diagrama de afinidad, a partir de los 18 factores se comprimieron en 5 factores fundamentales para medir la capacidad de exportación: 1) marketing, 2) logística, 3) planificación, 4) talento humano y 5) finanzas.

Se enfatiza que las 157 variables lingüísticas que componen los 18 factores surgieron de un análisis exhaustivo y una discusión por un equipo de expertos, después de evaluar la primera propuesta de 250 variables se consigue un rango final de 157 variables. Un conjunto tan amplio de datos tiene un ingrediente alto de imprecisión, así que se debe buscar una solución a través de conjuntos difusos.

El análisis se efectúa a través de 2 pasos:

En el primero se ponderan las 157 variables explicativas, a través de asignación de puntuaciones a cada una según su peso de acuerdo con la evaluación de expertos. Este procedimiento califica el peso de cada subfactor $F_{j i}(j=1 \ldots 18)$.

El segundo paso utiliza la calificación anterior para desarrollar una FIS para evaluar la capacidad de internacionalización. Las 18 entradas utilizadas como proxy de la capacidad de internacionalización se comprimen en 5 factores críticos de éxito mencionados anteriormente. Cada elemento 


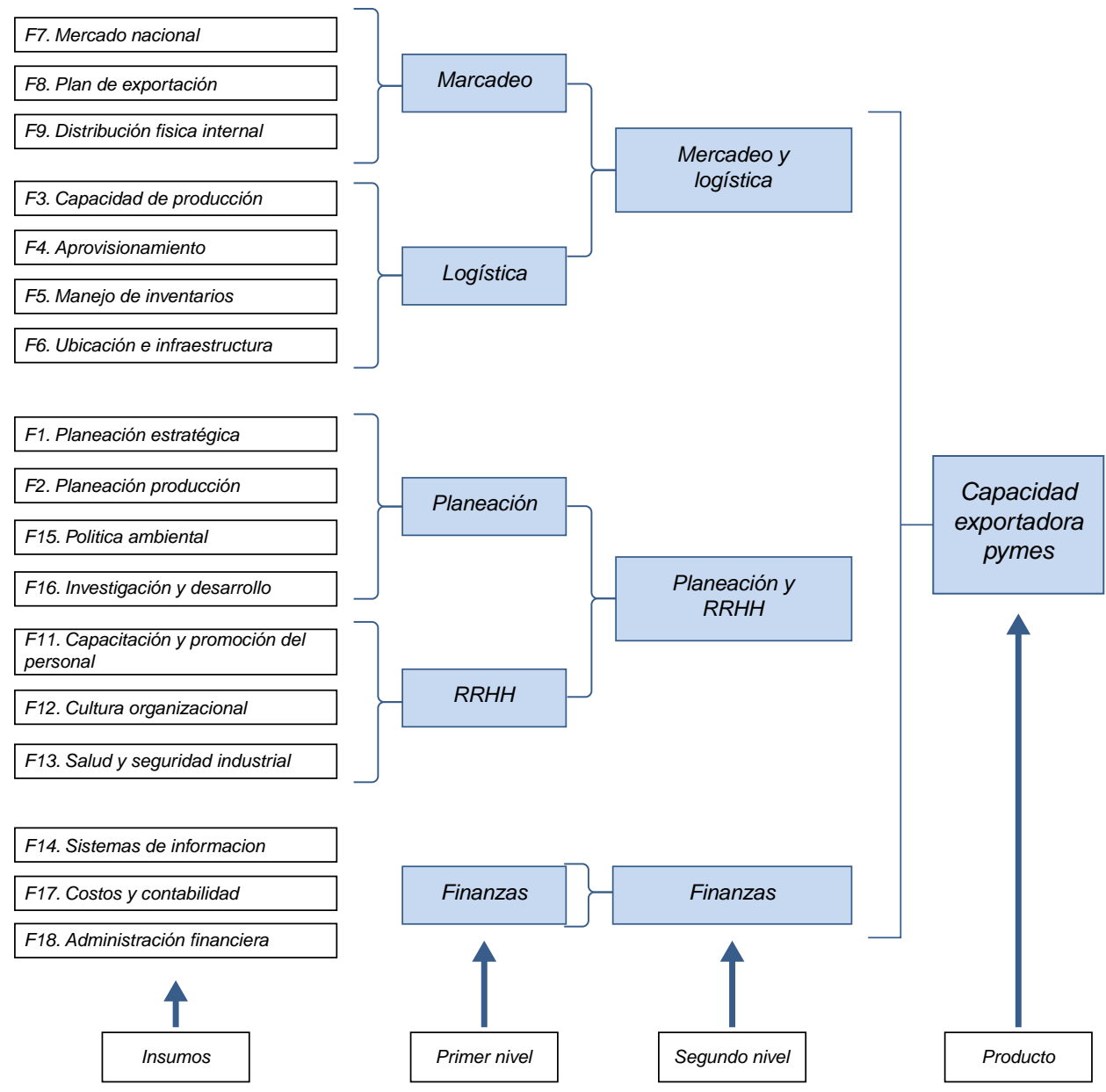

Figura 2. Subfactores para explicar capacidad de internacionalización.

Fuente: Elaboración propia.

representa un FIS de primer nivel en el sistema. Luego, las salidas de niveles bajos son las entradas del siguiente nivel. El siguiente paso recoge las 5 variables de nivel 1 y las transforma en 3 variables de segundo nivel: marketing y logística, planificación y talento humano, finanzas. Por último, el tercer nivel recoge toda la información reunida en los sistemas previos. El número total de sistemas difusos desarrollado fueron 8 , conectados entre ellos.

Los siguientes 4 conjuntos difusos son asignados a cada una de las entradas-salidas: fortaleza mayor (FMy), fortaleza menor (FMn), debilidad menor (DMn) y debilidad mayor (DMy), y se define un rango para cada variable entre 0 y 40, como representa en la figura 3.

Para construir la base de conocimientos o reglas borrosas del FIS aplicamos el procedimiento heurístico señalado en Medina (2010), que se utiliza en casos en los que se tienen en cuenta un gran número de variables de entrada. A continuación se resume el proceso: 


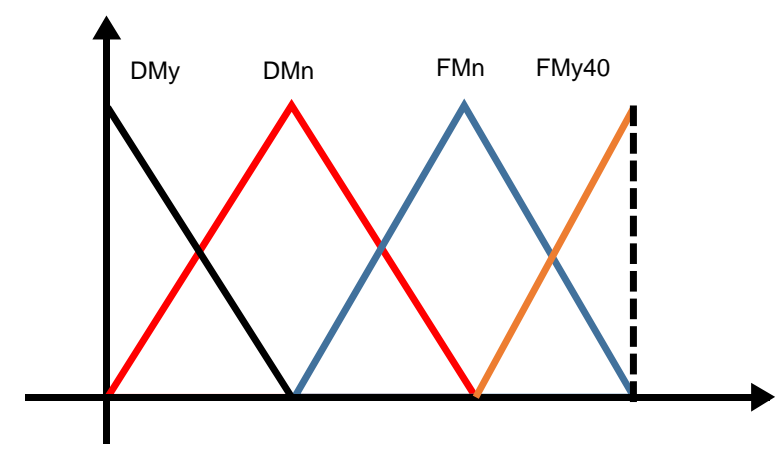

Figura 3. Conjuntos difusos para las variables de entrada y salida.

Fuente: Elaboración propia.

1) El primer paso es pensar cada entrada $X_{i}$, según el efecto que tiene en cada salida $Y_{j}$. Definir qué peso, $P_{i}$, se asigna a cada entrada (importancia relativa de la variable de entrada).

2) Donde, $P i \in[0,1, i=1, \ldots, n], n=$ variables de entrada del FIS. $J=1, \ldots, m, m$ es el conjunto difuso definido para la entrada $i$.

$$
\sum_{i=1}^{n} P_{i}=1
$$

3) Ponderación de la importancia de cada conjunto borroso (etiqueta lingüística) asignado a cada entrada $X_{i}$. Luego $C_{i j}$ es el peso asignado a cada conjunto borroso de entrada $X_{i}$ e indica el efecto sobre la salida $Y_{j}$.

$$
\sum_{j=1}^{m} C_{i j}=1 \text { para todo } i=1, \ldots, n
$$

4) Los expertos deben calificar cada escenario de la matriz de conocimiento, es decir, los expertos deben definir un conjunto de «reglas de referencia» para posteriormente validar la salida del FIS. En general, se recomienda definir escenarios extremos y medias, cuando el sistema es grande, para evaluar más fácilmente la calificación.

5) Reglas tipo Mamdani se expresan por: si $X_{1}$ es $A_{1}, X_{2}$ es $A_{2}, \ldots$ y $X_{k}$ es $A_{k}, Y$ entonces es $B$.

6) La puntuación para cada celda de la matriz de conocimiento se calcula por la siguiente relación:

$$
K_{i j}=\sum_{j=1}^{m} C_{i j} . P_{i} \quad \text { para } \mathrm{j}=1, \ldots, \mathrm{m}
$$

7) El paso siguiente consiste en correlacionar todas las puntuaciones en cada celda de la matriz de puntuación con los conjuntos difusos asignados a la variable de salida $Y_{j}$. Este procedimiento se lleva a cabo obteniendo el valor máximo y mínimo de la matriz de las puntuaciones, $K_{\max }$ y $K_{\min }$ respectivamente. $K_{\max }-K_{\min }$, lo que permite decidir el rango de resultados que puede tomar la variable de salida $Y_{j}$. Por lo tanto, los conjuntos difusos se deben asignar a este rango. 


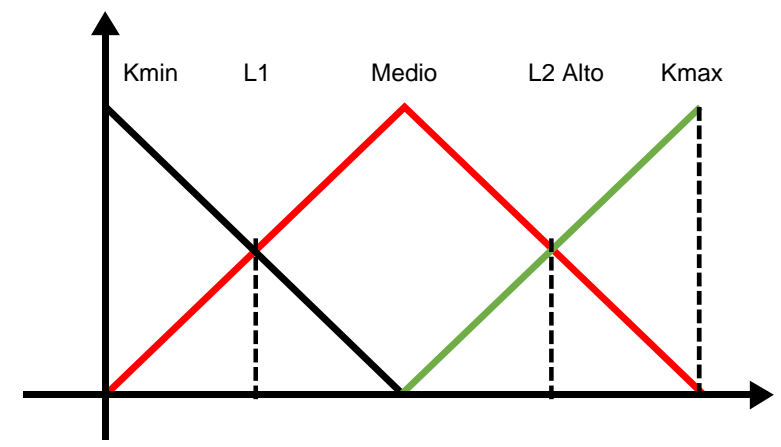

Figura 4. Conjunto de salida triangular $\mathrm{Yj}$.

Fuente: Elaboración propia.

La puntuación en la intersección de conjuntos difusos $\left(L_{1}\right.$ y $\left.L_{2}\right)$ decide el resultado desde el cual es asignado un conjunto borroso (bajo, medio, alto) a cada celda de la matriz (fig. 4).

La respuesta del sistema se puede ajustar cambiando la posición de los límites $L_{1}$ y $L_{2}$. La respuesta del sistema se ajusta a la matriz difusa de referencia, la figura 4 (compara la puntuación de cada celda con la puntuación de la intersección) exhibe las reglas previamente calificadas por los expertos. Este procedimiento permite conseguir la matriz de reglas borrosas.

Finalmente, la aprobación debería empezar con la matriz de reglas borrosas propuesta en el paso anterior para ajustar el sistema. Todas las celdas son evaluadas por expertos. Primero se requiere verificar que las «reglas de referencia»son definidas. Después, se debe verificar que todas las demás reglas propuestas sean consistentes, si no, los límites $L_{1}$ y $L_{2}$ deben moverse para ajustar la respuesta del sistema.

Después de aplicar el procedimiento, tenemos las matrices de reglas borrosas para cada sistema difuso (8 FIS, 8 matrices de reglas). La figura 5 muestra la matriz de reglas borrosas de tercer nivel que califican la capacidad de internacionalización de las pymes y se utilizaron como variables de entrada: marketing y logística, planificación y talento humano y finanzas con 64 reglas $(4 \times 4 \times$ 4). Las reglas se expresan como: si «marketing y logística» es «DMn» y «planificación y recursos humanos» es «FMn» y «Finanzas» es «DMn» entonces «la capacidad de internacionalización» es $« D M n »$.

\section{Modelo de aprobación}

Esta sección presenta la aprobación y consistencia del modelo. Se elabora a través de las sesiones de trabajo de expertos. Se contrastó el juicio de expertos versus el modelo simulado, verificando su funcionalidad y comparando con los resultados proyectados. Se consideran 3 escenarios, ordenados como casos: optimista, normal y pesimista, de acuerdo con los expertos, luego se comprueba que la salida de los sistemas sea consistente en cada caso.

El sistema FIS ajusta tanto las reglas if-then como el cambio de posición de los conjuntos difusos en los rangos definidos. A continuación, se busca una salida consistente con el juicio de expertos para la capacidad de internacionalización de las pymes (ECS). El grupo de expertos, consultores y empresarios, que fueron entrevistados y colaboraron con la normalización, son miembros de ACOPI, con experiencia en los mercados internacionales. 


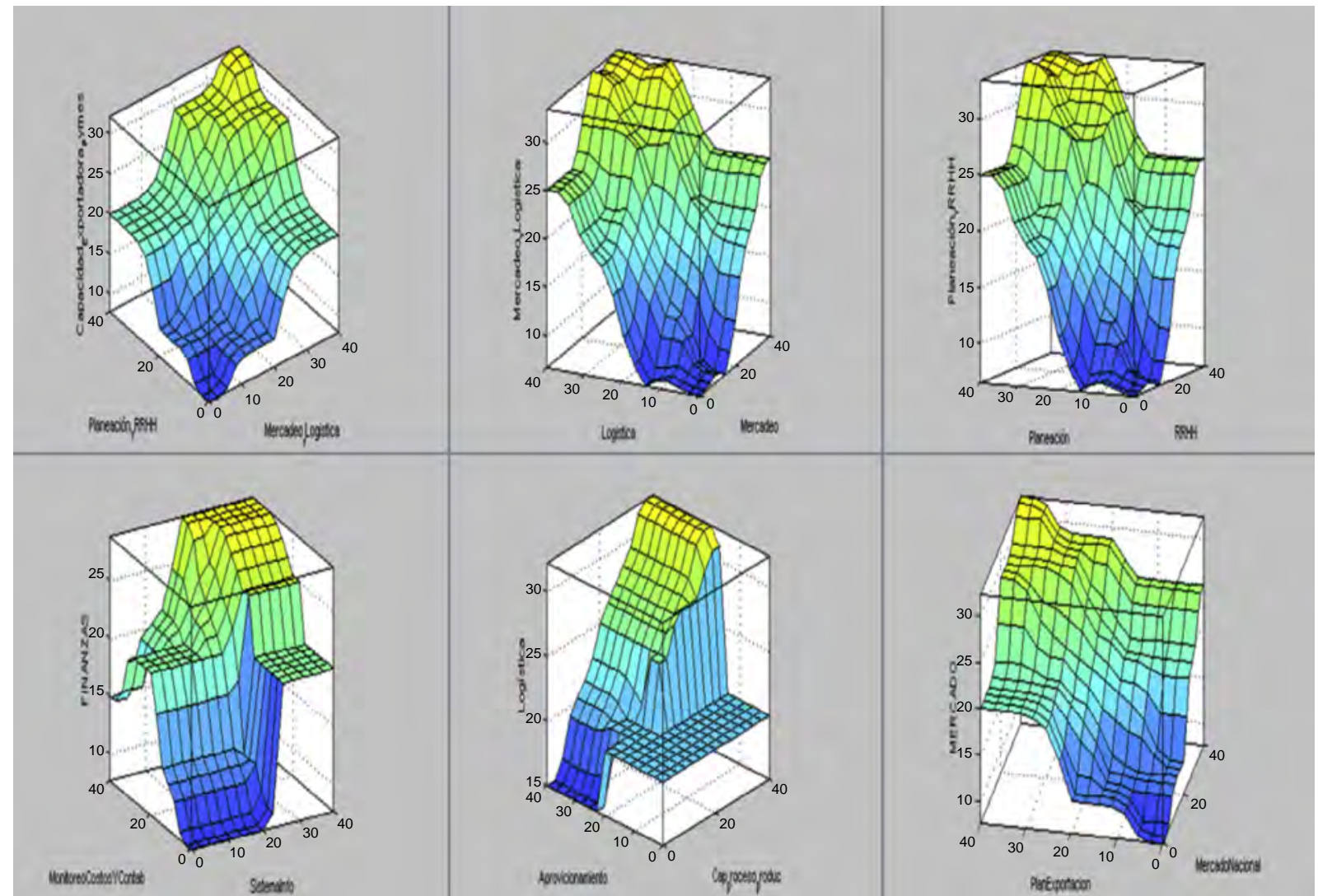

Figura 5. Gráficos de las variables de nivel 1.

Fuente: Elaboración propia utilizando el software Matlab. 

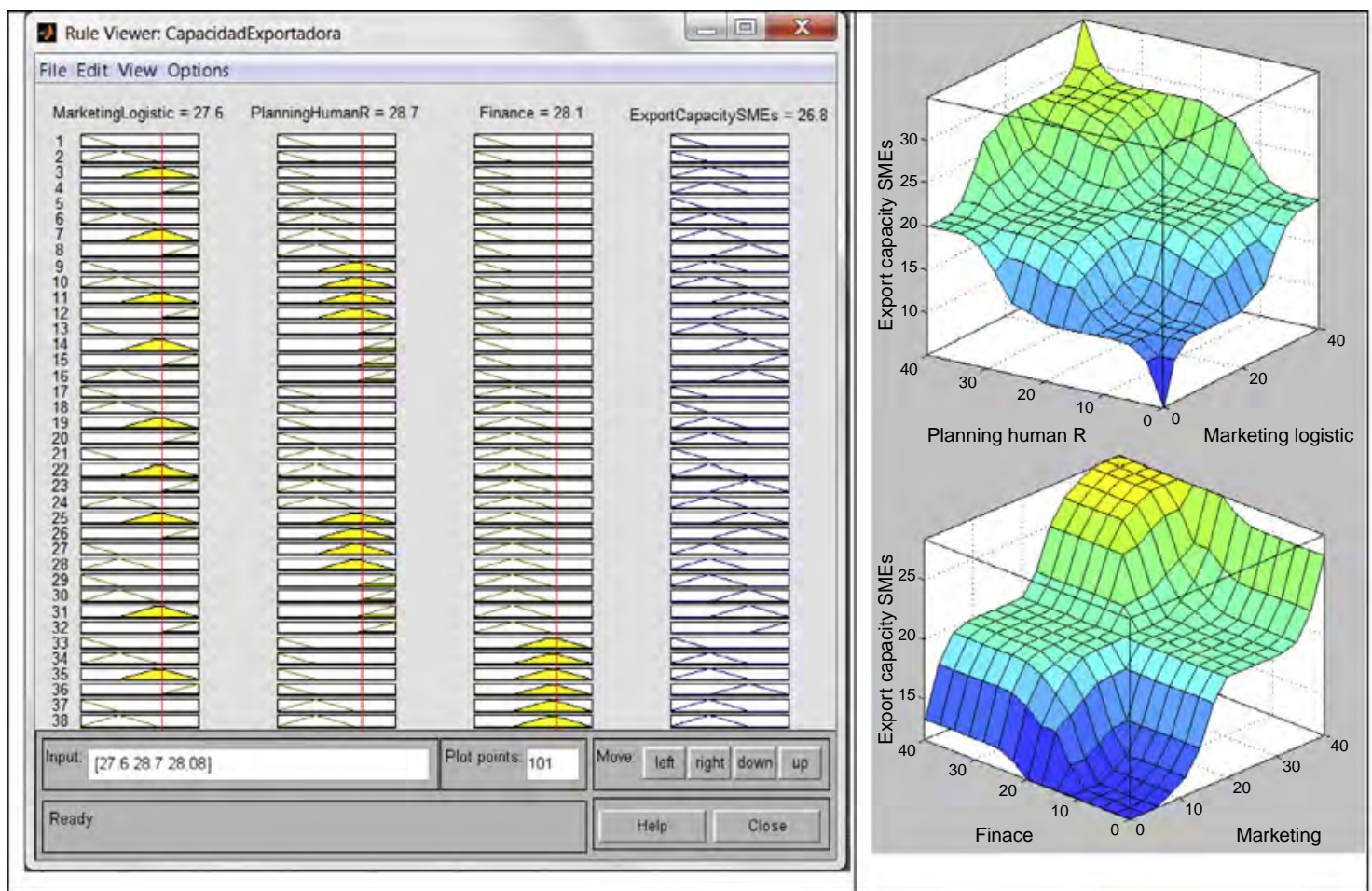

Figura 6. Visor de reglas y superficie difusa.Fuente: Elaboración propia utilizando el software Matlab. 
Tabla 1

Matriz de reglas difusas

\begin{tabular}{|c|c|c|c|c|c|c|c|c|c|c|c|c|c|}
\hline & \multicolumn{7}{|c|}{ Variables de nivel I } & & & & & & \\
\hline & \multicolumn{7}{|c|}{$\begin{array}{c}\text { Mercado } \\
0.3\end{array}$} & & & & & & \\
\hline & $\begin{array}{c}\begin{array}{c}\text { Mercado } \\
\text { nacional } \\
0.4\end{array} \\
\end{array}$ & & $\begin{array}{c}\begin{array}{c}\text { Plan de } \\
\text { exportación }\end{array} \\
0.3 \\
\end{array}$ & $\begin{array}{c}\text { Debilidad } \\
\text { magoot } \\
\text { of }\end{array}$ & $\begin{array}{l}\text { Debilidad } \\
\text { menor } \\
20 \%\end{array}$ & $\begin{array}{c}\text { Fortaleza } \\
\text { menor } \\
30 \%\end{array}$ & $\begin{array}{c}\text { Aacional } \\
\text { Fortaleza } \\
\text { magot } \\
\text { ser }\end{array}$ & & & & & & \\
\hline \multirow{4}{*}{$\begin{array}{l}10 \% \\
200 x\end{array}$} & \multirow{4}{*}{$\begin{array}{l}\text { Debilidad mayot } \\
\text { Debilidad menor } \\
\text { Fortulezamenor } \\
\text { Fortuleza magor }\end{array}$} & \multirow{4}{*}{$\begin{array}{l}10 \% \\
10 \% \\
10 \% \\
10 \%\end{array}$} & \multirow{4}{*}{$\begin{array}{l}\text { Debildid mayor } \\
\text { Debïdad mayor } \\
\text { Debildid mayor } \\
\text { Debisdad magor }\end{array}$} & 0.1 & 0,13 & 0.16 & 0.19 & DMs & $\mathrm{DM}_{8}$ & DM & $\mathrm{DMn}$ & $\max$ & (4,4 \\
\hline & & & & 0,14 & 0,17 & 0.2 & 0.23 & $\mathrm{OM}_{3}$ & $D M_{y}$ & $\mathrm{OMn}$ & $\mathrm{DMn}$ & $\min$ & 0.1 \\
\hline & & & & 0.13 & 021 & 0.24 & 0.27 & $\mathrm{Dam}$ & $\mathrm{DMn}$ & $\mathrm{OMm}$ & $\mathrm{FMn}$ & amplitud & 0,0750 \\
\hline & & & & 0,2 & 025 & 0,28 & ast & $\mathrm{OMn}$ & $F M n$ & $\mathrm{FMn}$ & FMn & \multirow[t]{2}{*}{ faetor } & \multirow[t]{2}{*}{$\lim \min$} \\
\hline \multirow{4}{*}{$30 \%}$. & Debilidad magor & \multirow{4}{*}{$\begin{array}{l}200 \% \\
20 \% \\
200 \% \\
200 \%\end{array}$} & \multirow{4}{*}{ 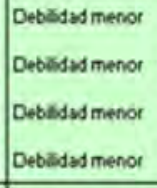 } & 0.0 & 0.16 & 0,19 & 0.22 & $\mathrm{OMM}_{\mathrm{O}}$ & $\mathrm{DMM}_{\mathrm{y}}$ & $\mathrm{OMn}$ & $\mathrm{DMn}$ & & \\
\hline & \multirow{3}{*}{$\begin{array}{l}\text { Deblidad menor } \\
\text { Fortaleza menor } \\
\text { Fortaleza mayor }\end{array}$} & & & $0.7 \pi$ & 0.2 & 0.23 & 0.26 & $\mathrm{OMM}_{\mathrm{s}}$ & DMn & $\mathrm{OMn}$ & FMn & \multirow{2}{*}{ factor } & \multirow{2}{*}{$\lim$ mas } \\
\hline & & & & 0,21 & 0.24 & 0.27 & 0,3 & $\mathrm{DMn}$ & DMn & FMn & $F M$ & & \\
\hline & & & & 0.25 & 0.28 & 0,31 & 0.34 & FMn & FMn & FMn & $F M$ & & \\
\hline \multirow{4}{*}{$\begin{array}{l}10 \% \\
20 \% x \\
30 \% \\
40 \times x\end{array}$} & \multirow{4}{*}{ 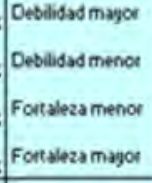 } & \multirow{4}{*}{$\begin{array}{l}30 \% \\
30 \% \\
30 \% \\
30 \%\end{array}$} & \multirow{4}{*}{$\begin{array}{l}\text { Fortaleza menot } \\
\text { Fortuleza menot } \\
\text { Fortaleza menor } \\
\text { Fortaleza menor }\end{array}$} & 0.16 & 0,19 & 0,22 & 0.25 & $D M_{3}$ & $\mathrm{DMn}$ & $\mathrm{OMn}$ & FMn & & \\
\hline & & & & 0.2 & 023 & 0.28 & 028 & $\mathrm{DMn}$ & DMD & FMn & $\mathrm{FMn}$ & & \\
\hline & & & & 0.24 & 0.27 & 0.3 & 0.33 & $\mathrm{DMn}$ & $\mathrm{FMn}$ & FMn & $\mathrm{FM}$ & & \\
\hline & & & & 0.28 & 0.31 & 0,34 & 0,37 & FMn & $\mathrm{FMn}$ & $F M_{y}$ & $F M_{y}$ & & \\
\hline \multirow{4}{*}{$\begin{array}{l}10 \% \\
20 \% 6 \\
30 \% \\
40 \% 6\end{array}$} & \multirow{4}{*}{$\begin{array}{l}\text { Debilidad mayot } \\
\text { Debdidad menot } \\
\text { Fortaleza menor } \\
\text { Fortaleza magor }\end{array}$} & \multirow{4}{*}{$\begin{array}{l}40 \% \\
40 \% \\
40 \% \\
40 \%\end{array}$} & \multirow{4}{*}{$\begin{array}{l}\text { Fortuleza magor } \\
\text { Fortaleza mayor } \\
\text { Fordaleza magor } \\
\text { Fortdeza magor }\end{array}$} & 0,19 & 0.22 & 0,25 & 0.28 & $\mathrm{DMn}$ & $\mathrm{DMn}$ & $\mathrm{FMn}$ & $F_{M}$ & & \\
\hline & & & & 0.23 & 0.26 & 0.29 & 0,32 & $\mathrm{DMn}$ & $\mathrm{FMn}$ & $\mathrm{FMn}$ & $\mathrm{FMn}$ & & \\
\hline & & & & 0.27 & 0.3 & 0,33 & 0,36 & $\mathrm{FMn}$ & $\mathrm{FMn}$ & $\mathrm{FM}_{3}$ & $\mathrm{FM}_{\mathrm{m}}$ & & \\
\hline & & & & 0.21 & 0.34 & 0.37 & 0.4 & $\mathrm{FMn}$ & FMy & FMy & $F M_{1}$ & & \\
\hline
\end{tabular}

$\mathrm{Kmac}=0,400$

$\mathrm{Kmin}=0,100$

Puntos de corte

Amplitud $=0,0750$

$\mathrm{L} 1=0,1750$

$\mathrm{L} 2=0,2500$

$\mathrm{L} 3=0,3250$

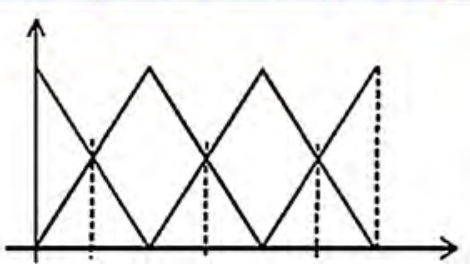

Fuente: Elaboración propia. 
Tabla 2

Resultados de nivel III

\begin{tabular}{|c|c|c|c|c|c|c|c|c|c|c|c|c|c|c|c|c|c|c|c|c|c|}
\hline \multicolumn{21}{|c|}{ Matriz de evaluación } & \\
\hline \multirow[t]{2}{*}{$2 \psi^{2}$} & Capacidad exporadora para pymes & \multicolumn{13}{|c|}{ Evaluación de expertos } & \multirow{2}{*}{ 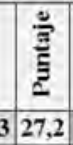 } & \multirow{2}{*}{$\begin{array}{c}\stackrel{8}{2} \\
2 \\
100 \%\end{array}$} & \multirow[t]{2}{*}{10} & \multirow[t]{2}{*}{20} & \multirow[t]{2}{*}{30} & \multirow{2}{*}{$\begin{array}{l}40 \\
\text { FMn }\end{array}$} & \\
\hline & Inputs & E1 & E2 & E3 & E4 & E5 & E6 & E7 & E8 & E9 & E10 & E11. & $\mid$ E12 & E13 & & & & & & & \\
\hline \multirow{4}{*}{$\begin{array}{l}\frac{8}{2} \\
\frac{2}{2} \\
\frac{2}{2} \\
\frac{2}{2}\end{array}$} & Mercado nacional & 30 & 32 & 29 & 31 & 34 & 28 & 31 & 30 & 29 & 31 & 31 & 31 & 28 & 30,4 & $30 \%$ & FMy & 0,24 & 0.76 & & \\
\hline & Plan de expartación & 27 & 28 & 27 & 25 & 24 & 26 & 25 & 27 & 28 & 27 & 26 & 26 & 27 & 26,4 & $35 \%$ & FMn & 0,12 & 0,88 & & \\
\hline & Distribución fisica internacional & 25 & 26 & 27 & 24 & 25 & 23 & 27 & 24 & 23 & 24 & 26 & 27 & 26 & 25,2 & $35 \%$ & FMn & 0,16 & 0,84 & & \\
\hline & & & & & & & & & & & & & & & 28,5 & $100 \%$ & OK & & & FMn & \\
\hline \multirow{4}{*}{ 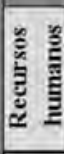 } & Estructura y organizaciān & 30 & 32 & 29 & 31 & 31 & 28 & 31 & 30 & 29 & $3 \mathrm{I}$ & 31 & 31 & 29 & 30,2 & $25 \%$ & FMy & 0,24 & 0,76 & & \\
\hline & Capacitación y promocion del persanal & 27 & 28 & 27 & 25 & 27 & 26 & 25 & 27 & 28 & 27 & 26 & 26 & 27 & 26,6 & $35 \%$ & FMn & 0,11 & 0,89 & & \\
\hline & Cultura organizacional & 25 & 29 & 28 & 30 & 29 & 27 & 29 & 26 & 27 & 29 & 28 & 27 & 28 & 27,8 & $20 \%$ & $\overline{F M n}$ & 0.07 & 0,93 & & \\
\hline & Salud y seguridad industrial & 30 & 32 & 29 & 31 & 34 & 28 & 31 & 30 & 29 & 31 & 31 & 31 & 25 & 30,2 & $20 \%$ & FMy & 0.25 & 0,75 & & \\
\hline \multirow{6}{*}{ 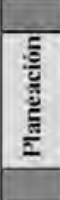 } & & & & & & & & & & & & & & & 29,2 & $100 \%$ & OK & & & FMn & \\
\hline & Proceso de plameamiento estratégico & 30 & 32 & 25 & 31 & 32 & 27 & 31 & 30 & 29 & 31 & 33 & 31 & 27 & 29,9 & $25 \%$ & FMn & 0,00 & 1,00 & & \\
\hline & Planificación y proceso de producción & 27 & 28 & 27 & 25 & 24 & 26 & 25 & 27 & 28 & 27 & 26 & 26 & 28 & 26,5 & $25 \%$ & FMn & 0,12 & 0,88 & & \\
\hline & Politica am biental de la empresa & 25 & 32 & 29 & 31 & 34 & 28 & 31 & 30 & 29 & 31 & 31 & 31 & 29 & 30,1 & $25 \%$ & FMy & 0,25 & 0,75 & & \\
\hline & Investigaciön y desartollo & 30 & 32 & 29 & 31 & 34 & 28 & 31 & 30 & 29 & 31 & 31 & 31 & 28 & 30,4 & $25 \%$ & FMy & 0,24 & 0,76 & & \\
\hline & & & & & & & & & & & & & & & 28,2 & $100 \%$ & $\mathrm{OK}$ & & & FMn & \\
\hline \multirow{5}{*}{ 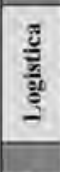 } & Capacidad del proceso de producción & 30 & 33 & 29 & 31 & 34 & 26 & 31 & 30 & 29 & 31 & 32 & 34 & 28 & 30,6 & $25 \%$ & FMy & 0.23 & 0,77 & & \\
\hline & Aprovisionamieruo & 27 & 28 & 27 & 25 & 24 & 26 & 25 & 27 & 28 & 27 & 26 & 26 & 26 & 26,3 & $25 \%$ & FMn & 0,12 & 0,88 & & \\
\hline & Manejo de inventarios & 29 & 32 & 29 & 31 & 34 & 28 & 31 & 30 & 29 & 31 & 31 & \begin{tabular}{|c|c|}
31 \\
\end{tabular} & 28 & 310,3 & $25 \%$ & FMy & 0.24 & 0,76 & & \\
\hline & Ubicacione infroestructura & 25 & 24 & 23 & 29 & 26 & 27 & 24 & 28 & 26 & 25 & \begin{tabular}{|l|l|l|l|l|l|l}
27 \\
\end{tabular} & 24 & 22 & 25,4 & $25 \%$ & FMn & 0,15 & 0,85 & & \\
\hline & & & & & & & & & & & & & & & 27,9 & $100 \%$ & OK & & & FMn & \\
\hline \multirow{3}{*}{ 宽 } & Sistemas informacion & 30 & 33 & 29 & 31 & 34 & 31 & 31 & 30 & 29 & 31 & 31 & 32 & 26 & 30,6 & $40 \%$ & FMy & 0,23 & 0,77 & & \\
\hline & Monitorea de costos y contubilidad & 27 & 28 & 27 & 25 & 24 & 29 & 25 & 27 & 28 & 27 & 26 & 29 & 25 & 26,7 & $35 \%$ & FMn & 0,11 & 0,89 & & \\
\hline & Administracion financiert & 25 & 24 & 23 & 24 & 26 & 27 & 26 & 27 & 24 & 23 & 27 & 26 & 28 & 25,4 & $25 \%$ & FMn & 0,15 & 0,85 & & \\
\hline
\end{tabular}

Fuente: Elaboración propia utilizando el software Matlab. 
Otra forma de confirmar el modelo es a través de las superficies difusas, que muestran las relaciones y la coherencia entre las entradas y salidas. Si los comportamientos no son consistentes se deben revisar las reglas de conocimiento, proponer el uso de otro conjunto borroso para las variables de entrada/salida o mover los conjuntos difusos en el rango definido para cada variable. Cuando las superficies son deformes, indican que el modelo es consistente y también señalan que no hay relación lineal entre las variables. La aplicación se desarrolla en Matlab. Un ejemplo de una superficie difusa se muestra en la figura 6.

La figura 7 muestra el editor de reglas y las superficies difusas para evaluar la capacidad de internacionalización de las pymes. Las superficies no marcan ninguna relación lineal entre variables, cuando las entradas incrementan su calificación (planificación y recursos humanos, marketing y logística y finanzas), entonces mejora la capacidad de exportación de la empresa. La evaluación permite identificar los aspectos que se deben mejorar y dónde es necesario tomar medidas administrativas para mejorar capacidad de exportación. Las calificaciones de planificación y recursos humanos $=28.7$, marketing y logística $=27.6$ y finanzas $=28.1$ dan como resultado una calificación para la capacidad de exportación de 26.7 que equivale a FMn. Se obtiene esta calificación después de evaluar los anteriores sistemas difusos (sistemas de primer y segundo nivel) Tabla 1.

Después de verificar la consistencia del algoritmo, un ajuste y aplicación experimental se lleva a cabo con el método propuesto. Los entrevistados identificaron varios beneficios en esta herramienta para el diagnóstico de ECS, así como el impacto de su aplicación en cualquier empresa del sector de manufactura. Por tanto, el enfoque del modelo fue aceptado por los encuestados, y se destacó el proceso de aprendizaje. Se infiere que las empresas del sector textil/confección requieren diseñar y cumplir con los programas, proyectos y estrategias para fortalecer la capacidad de innovar y competir para lograr el éxito en la región. Este examen se realiza en 13 empresas piloto miembros de ACOPI.

El ejercicio de agregación de resultados se reporta en la figura 8. Las evaluaciones se ponderaron según el peso dado a cada elemento, luego se calcula el valor promedio para la capacidad de internacionalización. Las columnas 3 a la 15 presentan la evaluación de los expertos, la columna 16 muestra el valor promedio y la columna 17 contiene el peso para cada entrada. Por ejemplo, el valor obtenido por el factor marketing es 27.2, y es el resultado de la suma ponderada de cada factor secundario de la siguiente manera: $30.4 \times 0.30+26.4 \times 0.35+25.2 \times 0.35=27.2$, que coinciden con el conjunto difuso FMn, según el juicio de expertos. Si se repite el procedimiento para cada factor, se obtiene un valor de 26.7 como calificación final de la capacidad de internacionalización, el cual coincide con una FMn. Este resultado es coherente con los resultados del modelo FIS (fig. 7) Tabla 2.

\section{Conclusiones}

En este trabajo se propone un marco lógico que integra variables lingüísticas para definir la capacidad de internacionalización como talento humano, infraestructura, estrategias de innovación, acuerdos de libre comercio, finanzas y estrategias de marketing; se construyó un FIS para modelar los determinantes del éxito de internacionalización considerando las variables lingüísticas — cualitativas y cuantitativas — que se consideran más relevantes para explicar cada factor considerado. El modelo propuesto se aplica como caso de estudio para una prueba piloto de 13 empresas del sector textil/confección en Medellín (Colombia).

En la ejecución del modelo se obtiene un valor particular de 26.7 para tales habilidades. El resultado obtenido confirma la hipótesis de que las capacidades y recursos de que dispone 
actualmente el sector analizado no son suficientes para una inserción exitosa en el mercado internacional y, lo más importante, especifica los factores y variables sobre los que intervenir para mejorar la capacidad de exportación. La evaluación de las variables planteadas en el estudio arrojó unos datos coherentes, confiables y validados que dan cuenta de la realidad del sector, con relación a la implementación de una metodología para evidenciar la capacidad de exportación. De acuerdo con el nivel de desarrollo de la unidad de I + D, esta se ubica como fortaleza menor con un valor de 24.97; se recomienda mejorar la variable mediante la adopción de un proceso formal de investigación de materias primas y procesos para convertir esta fortaleza menor en una fortaleza mayor. La variable sistemas de información presenta un valor nítido de 26.4, ubicándose como FMn, lo que implica que está cerca de convertirse en una DMn, razón que invita a su mejoramiento mediante la adopción de sistemas de información para las empresas que satisfagan los requerimientos funcionales de información de la gerencia general y de todos los departamentos, en forma oportuna y confiable. Igualmente, se precisa actualizar la empresa en materia de nuevos desarrollos en programas y equipos de cómputo, así como en la capacitación del personal para su manejo. El subfactor gestión ambiental se ubica con 27.6 como una FMn: se puede convertir en una FMy, mejorando la cultura y la estrategia de la compañía mediante la consideración de aspectos, impactos y riesgos ambientales en el proceso de planeación, con medidas referidas a considerar las regulaciones ambientales en el desarrollo de nuevos productos y servicios, o la ejecución de cambios en su infraestructura física. En cuanto al factor finanzas, ubicado con 29 como una FMn, es posible mejorar el sistema de contabilidad y costos y proveer información confiable, suficiente, oportuna y precisa para la toma de decisiones, así como contabilizar de forma periódica e incrementar la rotación de inventarios; igualmente, es necesario un sistema que permita definir los costos de la empresa en función de las características de los productos y procesos, y costear de forma diferente los productos de exportación.

Gerentes, dueños de empresa y expertos de ACOPI, con la aplicación del modelo, identificaron las fortalezas y debilidades para el proceso internacionalizador de las pymes de textil/confección, y subrayaron la importancia de aplicar el modelo en diferentes empresas de la industria manufacturera, debido a su flexibilidad y al amplio rango de variables evaluadas. Los resultados del modelo causaron preocupación entre los participantes acerca de la relevancia económica y la prioridad de las pymes en desarrollar e implementar programas, proyectos y estrategias para fortalecer las capacidades internas y enfrentar de forma adecuada la globalización y su innovación, además, de disminuir los efectos del libre comercio y la firma de tratados con diferentes países, como los Estados Unidos. Además, las pymes bajo estudio deben mejorar su modelo de internacionalización, incursionando en temas científicos y técnicos a través de alianzas con la red nacional de $\mathrm{I}+\mathrm{D}$.

El modelo planteado surge como una herramienta de gestión innovadora, vinculado con factores internos de la organización, sus recursos y habilidades. Se consideran un conjunto amplio de variables estratégicas, identificadas y relacionadas con rigor teórico y metodológico a través de la revisión de literatura, la asesoría de expertos, la percepción de los empresarios y la aplicación empírica de un sistema analítico para evaluar la capacidad de internacionalización de las pymes. Las variables seleccionadas pasaron por varios filtros propuestos por consultores, empresarios y expertos en comercio internacional.

Las variables para explicar la capacidad de internacionalización, utilizadas en este trabajo, son de carácter lingüístico y con un alto grado de dificultad para su cuantificación a través de valores numéricos. La teoría de la lógica difusa demuestra ser una concreta y potente herramienta para enfrentar este tipo de variables. Una vez que permite direccionar — con un enfoque hacia variables lingüísticas definidas-, y clasificar información de las variables y factores en una escala infinita 
de valores en el intervalo [0-1]. Por último, permite representar la pertenencia a una determinada categoría por medio de las funciones de membresía y resolver la pobre capacidad de expresión de la bipolaridad de la lógica clásica.

\section{Limitaciones del sistema de lógica difusa para modelar capacidades de internacionalización}

Las principales limitaciones para el uso de los FIS radican en la dificultad para identificar las relaciones entre las variables de entrada y salida. Este procedimiento toma tiempo y se basa en el trabajo de expertos y es más complejo cuando el número de variables de entrada se incrementa. En el caso particular de empresas del sector textil/confección, se resalta la falta de un registro continuo de información y la existencia de pocos estudios dirigidos hacia el desarrollo de planes a largo plazo. Del mismo modo, existe poca consistencia en los criterios definidos para exportar y se requiere de la participación y cooperación, tanto del clúster de la industria textil como del Estado.

\section{Línea de investigación futura}

El modelo descrito puede complementarse con estudios de capacidad de gestión administrativa y tecnológica, así como también con el análisis de recursos externos para un claro enfoque en el mercado internacional. Según lo descrito, la versatilidad de los sistemas de lógica difusa presenta un importante espectro de servicios para el sector académico y empresarial vinculado con propuestas innovadoras para direccionar temáticas con un alto grado de inexactitud.

\section{Anexo. Variables explicativas}

\begin{tabular}{ll}
\hline & 1. PLANEAMIENTO ESTRATÉGICO \\
F1. PROCESO DE PLANEAMIENTO ESTRATÉGICO \\
V1 & La empresa ha realizado un proceso de planeamiento estratégico en los últimos 2 años \\
V2 & La empresa tiene una estrategia básica de negocios escrita y conocida por todos los que deben ejecutarla \\
V3 & La empresa tiene como política para la toma de decisiones involucrar a las personas responsables por su \\
& ejecución y cumplimiento \\
V4 & El planeamiento estratégico es el resultado de un trabajo en equipo y participan en su elaboración quienes \\
& Son responsables por su ejecución y cumplimiento \\
V5 & Se definen objetivos específicos, cuantificables y medibles, junto con un plazo de tiempo definido para su \\
V6 & ejecución, por parte de las personas responsables del área o departamento involucrados \\
V7 planear se desarrolla un análisis DOFA (Debilidades, Oportunidades, Fortalezas y Amenazas) para la \\
empresa y el sector donde esta funciona, con la adecuada participación de las áreas
\end{tabular}




\section{LOGÍSTICA Y OPERACIONES}

\section{F2. PLANIFICACIÓN Y PROCESO DE PRODUCCIÓN}

El proceso de producción de la empresa es adecuado para fabricar productos con calidad y costos competitivos

La empresa tiene un programa escrito y detallado de adquisición de maquinaria y tecnología para ser ejecutado en el futuro previsible

El proceso de producción es suficientemente flexible para permitir cambios en los productos, en función de satisfacer las necesidades de los clientes

El planeamiento de la producción está basado en pronósticos de ventas, desprendidos de estudios de mercado

La empresa tiene medidas de control para el flujo de producción (desde la recepción de los materiales hasta la entrega de los productos terminados) para conocer el estado y avance de las órdenes de producción

La empresa evalúa con frecuencia la posibilidad de comprar materiales semiprocesados, así como de producir aquellos que provienen de proveedores (integración vertical o subcontratación)

La maquinaria y la tecnología de la empresa le permiten fabricar productos competitivos, a nivel nacional, en calidad y precio

La empresa implementa buenas prácticas de manufactura

Existe un plan de mantenimiento preventivo

El personal se capacita en buenas prácticas de manufactura

Existe una programación y un plan de compras

\section{F3. CAPACIDAD DEL PROCESO DE PRODUCCIÓN}

La empresa conoce la capacidad de producción de su maquinaria y equipo por cada línea de producción y de su recurso humano y define el rango deseado de su utilización

La empresa tiene planes de contingencia para ampliar su capacidad de producción más allá de su potencial actual para responder a una demanda superior a su capacidad de producción

Se realiza un programa de mantenimiento preventivo a todos los equipos y maquinaria y los resultados son debidamente documentados

La empresa mantiene un inventario de partes y repuestos claves para equipos críticos

Los operarios de los equipos participan en su mantenimiento

La empresa establece su programa de mantenimiento bajo el concepto del mantenimiento predictivo

La empresa establece su programa de mantenimiento bajo el concepto del mantenimiento total productivo (MTP)

La empresa tiene seguro contra incendio y otras calamidades devastadoras, así como de un lucro cesante adecuado

El personal está activamente involucrado en el logro de los objetivos de la empresa, así como en los cambios que demanda la implementación de la estrategia

Existe un sistema de rendición de cuentas periódico

El planeamiento estratégico da las pautas para la asignación general de recursos en cada área del negocio de la empresa, con un seguimiento efectivo

Es frecuente la programación de horas extras para cumplir con los pedidos

\section{F4. APROVISIONAMIENTO}

Existen criterios formales para la planificación de la compra de materias primas, materiales y repuestos (pronósticos de venta, disponibilidad, plazo de entrega, etc.)

Existe un sistema de abastecimiento flexible y eficiente que satisface las necesidades de la planta La empresa tiene un plan de contingencia para proveerse de materias primas críticas, tecnologías críticas y personal crítico que garanticen el normal cumplimiento de sus compromisos comerciales

En general, el criterio usado para seleccionar proveedores de materia prima y materiales es, en su orden, (1) calidad, (2) servicio, (3) precio y (4) condiciones de pago

\section{F5. MANEJO DE INVENTARIOS}

Como resultado de negociaciones con los proveedores se han programado las entregas de materias primas para mantener el inventario en un nivel óptimo según necesidades

Hay un nivel óptimo de inventario de materias primas, trabajo en proceso y producto terminado para reducir las pérdidas originadas en el mal manejo

El sistema de almacenamiento y administración de inventarios (materia prima, suministros y producto terminado) garantiza adecuados niveles de rotación, uso y control de estos 
V42 Con periodicidad programada se compara el inventario físico de materia prima, materiales y producto terminado con el inventario llevado en el kardex (tarjetas o electrónico)

\section{F6. UBICACIÓN E INFRAESTRUCTURA}

V44 distribución del producto terminado La infraestructura e instalaciones de la planta son adecuadas para atender sus necesidades actuales y futuras

La infraestructura de logística y la de acceso son adecuadas para atender sus necesidades actuales y futuras

La infraestructura e instalaciones del personal garantizan las necesidades actuales y futuras del personal

\section{COMERCIALIZACIÓN}

\section{F7. MERCADO NACIONAL}

El proceso de planeamiento genera un plan de mercadeo anual, escrito y detallado, con responsables e índices de gestión claramente definidos

La empresa tiene claramente definido su mercado objetivo, sus estrategias de penetración, posicionamiento y comercialización

La empresa conoce los segmentos del mercado en que compite, su participación, crecimiento y rentabilidad y desarrolla estrategias comerciales escritas para cada uno de ellos

La empresa establece objetivos o cuotas de venta, de recaudo y de consecución de clientes nuevos a cada uno de sus vendedores y controla su cumplimiento

La empresa dispone de información de sus competidores (en cuanto a reputación, calidad de sus productos y servicios, fuerza de ventas y precios)

Las estrategias, objetivos y precios de la empresa están determinados con base en el conocimiento de sus costos, la oferta, la demanda y la situación competitiva

En los últimos 2 años, los productos nuevos (menores de 3 años) han generado un porcentaje importante de las ventas y de las utilidades totales de la empresa

Los recursos asignados al mercadeo (material publicitario, promociones, etc.) son adecuados y se usan de manera eficiente

La empresa dispone de un sistema de información y análisis que le permite obtener información actualizada sobre sus clientes, sus necesidades y los factores que guían sus decisiones de compra La empresa evalúa periódicamente sus mecanismos de promoción, sistemas de información de mercados y seguimiento de tendencias

\section{Existe una programación y un plan de compras}

El personal que tiene contacto con el cliente es consciente de sus responsabilidades y tiene suficiente autonomía para atender adecuadamente sus necesidades

La empresa tiene un sistema de investigación que le permita conocer el nivel de satisfacción del cliente, lo documenta y toma acciones con base en su análisis

La empresa dispone de catálogos y especificaciones técnicas de sus productos

La empresa posee una fuerza de ventas capacitada, motivada y competente que apoya el cumplimiento de los objetivos de la empresa

La empresa ha desarrollado un sistema eficiente de distribución que permite llevar sus productos a sus clientes cuando y donde ellos los necesitan

La empresa prefiere contratar vendedores con vínculo laboral en lugar de independientes sin vínculo laboral

\section{F8. MERCADO EXPORTACIÓN: PLAN DE EXPORTACIÓN}

La empresa tiene un plan anual de exportación, escrito y detallado

La empresa planea exportar un volumen importante en los próximos 2 años

La empresa diseña sus productos para la exportación en forma diferente a como diseña para el mercado nacional

La empresa conoce y cumple las normas de calidad y de identificación (rotulación) que deben cumplir sus productos de exportación

El departamento responsable del desarrollo de nuevos productos cuenta con un presupuesto formal y adecuado, el equipo requerido y el personal calificado para realizar eficientemente su trabajo La empresa tiene un procedimiento para investigar, analizar, elegir y explotar nuevos mercados de exportación 
En los últimos 2 años, las exportaciones han generado un porcentaje importante de las ventas y de las utilidades totales de la empresa

La empresa tiene un conocimiento claro de la competencia y del entorno competitivo en los mercados de exportación seleccionados

La empresa hace un seguimiento a sus exportaciones para medir el nivel de satisfacción del cliente y asegurar su recompra

Se dispone de catálogos de productos, folletos publicitarios y especificaciones técnicas para el mercado de exportación (preferiblemente en inglés o en el idioma del mercado de destino)

La empresa tiene un procedimiento para investigar, analizar, elegir y explotar nuevos mercados de exportación

En los últimos 2 años, las exportaciones han generado un porcentaje importante de las ventas y de las utilidades totales de la empresa

La empresa tiene un conocimiento claro de la competencia y del entorno competitivo en los mercados de exportación seleccionados

La empresa hace un seguimiento a sus exportaciones para medir el nivel de satisfacción del cliente y asegurar su recompra

Se dispone de catálogos de productos, folletos publicitarios y especificaciones técnicas para el mercado de exportación (preferiblemente en inglés o en el idioma del mercado de destino)

La empresa hace un seguimiento a sus exportaciones para medir el nivel de satisfacción del cliente y asegurar su recompra

Se dispone de catálogos de productos, folletos publicitarios y especificaciones técnicas para el mercado de exportación (preferiblemente en inglés o en el idioma del mercado de destino)

\section{F9. MERCADO EXPORTACIÓN: DISTRIBUCIÓN FÍSICA INTERNACIONAL}

La empresa conoce el manejo de la distribución física internacional, sus costos y su impacto en el precio de exportación

La empresa cumple con los requisitos de tiempo de entrega al cliente internacional

La empresa toma las precauciones suficientes para evitar la introducción de drogas ilícitas en su mercancía de exportación

La empresa conoce sus costos, los precios de su competencia internacional y las condiciones generales

del sector que le permitan negociar con seguridad con sus clientes, canales de distribución y otros actores

La empresa ha participado en misiones comerciales a otros países

La empresa ha participado como observador en ferias internacionales (relacionadas con el negocio) en los últimos 2 años

La empresa ha participado como expositor en ferias internacionales (relacionadas con el negocio) en los últimos 2 años

La empresa tiene personal adecuadamente familiarizado con sus productos y procesos y adicionalmente domina el inglés

La empresa ha enviado sus funcionarios a misiones en países de América Latina

La empresa ha programado ser expositor en ferias internacionales (relacionadas con el negocio) en los próximos 2 años

La empresa ha programado participar como observador en ferias internacionales (relacionadas con el negocio) en los próximos 2 años

La empresa ha participado en misiones comerciales a otros países

La empresa ha participado como observador en ferias internacionales (relacionadas con el negocio) en los últimos 2 años

La empresa ha participado como expositor en ferias internacionales (relacionadas con el negocio) en los últimos 2 años

La empresa tiene personal adecuadamente familiarizado con sus productos y procesos y adicionalmente domina el inglés

La Empresa ha enviado sus funcionarios a misiones en países de América Latina

La empresa ha programado ser expositor en ferias internacionales (relacionadas con el negocio) en los próximos 2 años

La empresa ha programado participar como observador en ferias internacionales (relacionadas con el negocio) en los próximos 2 años

La empresa ha programado participar en misiones comerciales a otros países en los próximos 2 años 


\section{RECURSOS HUMANOS}

\section{F10. RECURSOS HUMANOS}

V100 La empresa tiene un organigrama escrito e implantado donde las líneas de autoridad y responsabilidad están claramente definidas

V101 La empresa tiene políticas y manuales de procedimientos escritos, conocidos y acatados por todo el personal

V103 La empresa cumple con todos los requisitos legales vigentes (ISS u otra EPS, SENA, cajas de compensación, reglamento de trabajo, reglamento de seguridad industrial, etc.)

V104 La empresa tiene por escrito los manuales de funciones, perfiles, planes de capacitación y carrera administrativa

\section{F11. CAPACITACIÓN Y PROMOCIÓN DEL PERSONAL}

La empresa tiene un programa definido para la capacitación de todo su personal y al personal nuevo se le da una inducción a la empresa

V106 Las habilidades personales, las calificaciones, el deseo de superación, la creatividad y la productividad son criterios claves para la remuneración y promoción del personal, así como para la definición de la escala salarial

V107 La empresa tiene por escrito los manuales de funciones, perfiles, planes de capacitación y carrera administrativa

\section{F12. CULTURA ORGANIZACIONAL}

La empresa realiza frecuentemente actividades sociales, recreativas y deportivas y busca vincular a la familia del trabajador en estos eventos

\section{F13. SALUD Y SEGURIDAD INDUSTRIAL}

V113 La empresa tiene un programa de salud ocupacional implementado (plan de prevención de enfermedades ocupacionales, seguridad laboral, planes de emergencia, etc.) cuando ocurren y toma acciones preventivas y correctivas

V116 La empresa lleva un registro de ausentismo ocasionado por enfermedades, accidentes de trabajo y otras causas

\section{SISTEMAS DE INFORMACIÓN F14. SISTEMAS INFORMACIÓN}

V117 El sistema de información de la empresa está diseñado para satisfacer los requerimientos funcionales de información de la gerencia general y de todos los departamentos en forma oportuna y confiable

V118 La empresa está actualizada en materia de nuevos desarrollos en programas y equipos de cómputo y tiene el personal capacitado para manejarlos

V119 El diseño técnico y funcional del sistema responde a las necesidades de información de la empresa y es óptimo con relación al tiempo de proceso y seguridad

V120 Se generan y archivan adecuadamente los documentos de soporte (órdenes de producción, entradas y salidas de almacén, comprobantes de egreso, recibos de caja, facturas, etc.) en las diferentes áreas de la empresa

V121 La captura de información genera operaciones simultáneas en las diferentes áreas de la empresa evitando la doble digitación de las transacciones en los diferentes sistemas

V122 Como política, la empresa realiza sistemáticamente copias de respaldo (backups) de sus archivos más importantes y los almacena en sitios seguros

V123 Existen procedimientos de contingencia, manuales o automatizados, en caso de pérdidas de fluido eléctrico o fallas en el equipo de proceso

V124 La información generada por el sistema es confiable, oportuna, clara y útil y es usada para la toma de decisiones

V125 La gerencia ha definido reportes que indiquen el tipo de datos requeridos para el proceso de toma de decisiones 


\section{GESTIÓN AMBIENTAL}

\section{F15. POLÍTICA AMBIENTAL DE LA EMPRESA}

En el diseño de la planta, la empresa tuvo en cuenta las regulaciones ambientales y el bienestar de sus trabajadores

V127 La empresa conoce las normas ambientales que la controlan y establece los procedimientos y procesos para cumplirlas

La cultura y la estrategia de la compañía involucran aspectos, impactos y riesgos ambientales

La empresa mide el desempeño ambiental frente a metas y estándares preacordados

La empresa considera las regulaciones ambientales cuando desarrolla nuevos productos y servicios, o realiza cambios en su infraestructura física

V131 Para la selección, instalación, operación y mantenimiento de los equipos se realizaron consideraciones ambientales, además de los aspectos técnicos y económicos

V132 Se definen y documentan las tareas, responsabilidades, competencias y procedimientos específicos que aseguren el cumplimiento de las normas ambientales, tanto internas como externas

V133 La empresa trata de minimizar el consumo de energía, agua y materias primas contaminantes mediante la mejora de sus procesos productivos, el reciclaje, la sustitución de insumos, el mantenimiento preventivo y el uso de otras tecnologías

V134 La empresa ha medido la cuantía del desperdicio, sabe en qué etapa del proceso es generado y ha formulado planes para reducirlo

\section{INVESTIGACIÓN Y DESARROLLO}

\section{F16. INVESTIGACIÓN Y DESARROLLO}

V135 La innovación es incorporada en los diferentes procesos de la empresa y se considera de vital importancia para su supervivencia

Existe un proceso formal de investigación de nuevas materias primas y procesos de producción

La empresa dispone de un programa de investigación y seguimiento a las tecnologías claves para sus diferentes negocios

La empresa tiene un programa escrito y detallado de adquisición de equipo, tecnología y modernización de sus procesos de producción

Existe un plan de mejoramiento continuo con indicadores y seguimiento

Cada año se incrementa el portafolio de productos como resultado de estudios de investigación de mercados

Un porcentaje de las ventas corresponde a productos desarrollados en los 2 últimos años

Un número importante de empleados de producción participan de programas de reinducción,

entrenamiento y capacitación

\section{CONTABILIDAD Y FINANZAS}

\section{F17. MONITOREO DE COSTOS Y CONTABILIDAD}

V142 El sistema de contabilidad y costos provee información confiable, suficiente, oportuna y precisa para la toma de decisiones períodos de antigüedad

Los productos de exportación se costean en forma diferente que los productos que van al mercado doméstico

V148 El sistema de costos de la compañía puede costear rápidamente pedidos, para el mercado nacional o internacional, con base en datos confiables

V149 Se comparan mensualmente los resultados financieros con los presupuestos, se analizan las variaciones y se toman acciones correctivas

V150 La empresa evalúa la utilidad de sus inversiones en equipo, otros activos fijos y en general de sus inversiones

V151 La empresa tiene una planeación financiera formal (presupuestos de ingresos y egresos, flujos de caja, razones financieras, punto de equilibrio, etc.)

V152 La empresa conoce la rentabilidad de cada producto o línea de producto 
V153 Los libros de actas, los registros de socios, las reformas de escrituras, la información para las superintendencias se encuentran al día y están debidamente archivados

V154 Se ha evaluado la inscripción de la empresa ante la Superintendencia de Industria y Comercio, Cámara de Comercio y la DIAN a la luz de su objeto social

V155 Se tiene claramente definido el calendario tributario de la empresa, con fechas definidas de entrega de declaraciones y otros documentos

V156 La empresa aplica los respectivos indicadores a sus declaraciones tributarias y se monitorean sus resultados

V157 La empresa tiene una planificación tributaria definida, conoce los montos aproximados por pagar en el período gravable de los diferentes impuestos, tasas y contribuciones

\section{Referencias}

Amit, R. y Schoemaker, P. (2012). Strategic assets and organizational rent. Strategic Management Journal, 14(1), $33-46$.

Aguirre, J. (2010). Metodología para medir y evaluar las capacidades tecnológicas de innovación aplicando sistemas de lógica difusa: caso fábricas de software. [tesis de maestría] Medellín (Colombia): Universidad Nacional de Colombia.

Alonso, A. (1994). El proceso de internacionalización de la empresa. Revista Información Comercial Española, 725, $127-143$.

Arango, D., Serna, C. y Pérez, G. (2012). La gestión de indicadores empresariales con lógica difusa para la toma de decisiones. Lámpsakos, 8, 47-53.

Barney, J. (1991). Firm resources and sustained competitive advantage. Journal of Management, 17(1.), 99-120.

Bellman, R. y Zadeh, L. (1970). Decision making in a fuzzy environment. Management Science, 17(4), 141-164.

Bisang, R., Fuchs, M. y Kosacoff, B. (1992). Internacionalización de empresas industriales argentinas. Desarrollo Económico, 323-356.

Björk, K. M. (2009). An analytical solution to a fuzzy economic order quantity problem. International Journal of Approximate Reasoning, 50(3), 485-493.

Blankenburg Holm, D. (1995). A network approach to foreign market entry. An interaction and network perspective. Business Marketing, 375-405.

Castro, F. (2007). Modelo de internacionalización para la empresa colombiana. Universidad Empresa, 6(12), 168-193.

Cejas-Montero, J. (2011). The compensatory fuzzy logic. Ingeniería Industrial, 32(2), 157-161.

Claver, E. y Quer, D. (2001). La dirección estratégica de la internacionalización de la empresa: propuesta de un marco teórico integrado. Revista Información Comercial Española, 794, 37-59.

Cruz, J. (2010). La internacionalización de las Pymes locales. Incae Business Review, 1(12), 52-59.

Darshan, K., Jagdev, S. y Om Pal, S. (2012). A fuzzy logic based decision support system for the evaluation of suppliers in supply chain management practices. Mathematical and Computer Modelling., 102(2), 289-301.

Dourra, H. y Siy, P. (2002). Investment using technical analysis and fuzzy logic. Fuzzy Set and Systems, 127, 221-240.

Escandón, D. y Hurtado, A. (2013). Internacionalización de empresas bajo la perspectiva de recursos y capacidades: caso sector autopartes. Cuadernos Latinoamericanos de Administración, IX(17), 72-85.

Gaur, A., Kumar, V. y Singh, D. (2014). Institutions, resources, and internationalization of emerging economy firms. Journal of World Business, 49, 12-20.

Gómez Suárez, Á., González, J., Lusa, E. y Osorio, J. (2006). Innovación y nuevas tecnologías en las pymes de Galicia. Sectores textil y conservero. Revista Galega de Economía, 15(2), 1-19.

Gutiérrez, A. y Heras, L. (2000). La proyección exterior de las empresas españolas: una contrastación empírica de la teoría gradualista de la internacionalización. Revista Información Comercial Española, 788, 7-18.

Hamel, G. y Prahalad, C. (1994). Competing for the future. Boston: Harvard Business School Press.

Huerta, P., Navas, L. J. y Almodóvar, P. (2004). La diversificación desde la teoría de recursos y capacidades. Cuadernos de Estudios Empresariales, 14, 87-104.

Inexmoda (2011). Datos de utilidad sobre el sector textil/confección de Colombia. [consultado 10 May 2014]. Disponible en: http://www.inexmoda.org.co/

Jang, J., Mizutani, E. y Sun, C. (1997). Neuro-fuzzy and soft computing: A computional approach to learning and machine intelligence. New Jersey: Prentice Hall. 
Johanson, J. y Mattson, L. (1988). Internationalization in industrial systems- a network approach. Strategies in Global Competition, 287-314.

Johanson, J. y Wiedersheim-Paul, F. (1975). The internationalization of the firm: Four Swedish cases. Journal of Management Studies, 12(3), 305-322.

Kaleka, A. (2002). Resources and capabilities driving competitive advantage in export markets: Guidelines for industrial exporters. Industrial Marketing Management, 31, 273-283.

Kasabov, N. (1998). Foundations of neural networks, fuzzy systems and knowledge engineering. United States: The MIT Press.

Kosko, B. (1994). Fuzzy systems as universal approximators. IEEE Transactions on Computers, 43(11), 1329-1333.

Lin, Y. y Wu, L. (2014). Exploring the role of dynamic capabilities in firm performance under the resource-based view framework. Journal of Business Research, 67(3), 407-413.

Mamdani, E. (1977). Application on fuzzy logic to approximate reasoning using linguistic synthesis. IEEE Transactions on computers, C26, 1182-1191.

Marchi, G., Vignola, M., Facchinetti, G. y Mastroleo, G. (2014). International market selection for small firms: A fuzzybased decision process. European Journal of Marketing, 48(11/12).

Martín del Brio, B. y Sanz, A. (2002). Redes neuronales y sistemas difusos. México, D.F: Alfaomega Grupo Editor.

Medina, S. (2006). Estado de la cuestión acerca del uso de la lógica difusa en problemas financieros. Cuadernos de Administración, 19(32), 195-223.

Medina, S. (2010). Modeling of operative risk using fuzzy expert systems. En Michael Glykas (Ed.), Fuzzy cognitive maps advances in theory, methodologies, tools and applications. Greece: Springer.

Medina, S. y Paniagua, G. (2008). Modelo de inferencia difuso para estudio de crédito. Dyna, 75(154), 215-229.

Mendoza, S. (2009). Sistema de lógica difusa. Una aplicación a la percepción empresarial. Revista Universidad \& Empresa, $17,252-270$.

Porter, M. (1985). Competitive advantage: Creating and sustaining superior perfomance. New York: Free Press.

Rajendra, A. y Priti, S. (2009). Knowledge-based systems. United States: Jones \& Bartlett Learning.

Rialp, A. (1999). Los enfoques micro-organizativos de la internacionalización de la empresa: una revisión y síntesis de la literatura. Revista Información Comercial Española, 781, 117-128.

Root, F. (1994). Entry strategies for international markets. San Francisco: Lexington Books.

Rush, H., Bessant, J. y Hobday, M. (2007). Assessing the technological capabilities of firms: Developing a policy tool. R\&D Management, 37(3), 221-236.

Sánchez, P., Herrera, F. y Herrera-Viedma, E. (2009). A fuzzy model to evaluate the suitability of installing an enterprise resource planning system. Information Sciences, 179(14), 2333-2341.

Santiso, J. (2007). La internacionalización de las empresas españolas: hitos y retos. Revista Información Comercial Española, 839, 89-102.

Serrano, J. (2010). Metodología para evaluar las capacidades de innovación tecnológica en una institución universitaria utilizando un sistema difuso. [tesis de maestría]. Universidad Nacional de Colombia: Medellín.

Teece, D. (2007). Explicating dynamic capabilities: The nature and microfoundations of (sustainable) enterprise performance. Strategic Management Journal, 28, 1319-1350.

Tallman, S. y Fladmoe-Lindquist, K. (2002). Internationalization, globalization, and capability-based strategy. California Management Review, 45(1), 116.

Thomas, M. y Araujo, L. (1985). Theories of export behavior: A critical analysis. European Journal of Marketing, 19(2), 42-52.

Thorelli, H. y Cavusgil, S. (1990). International marketing strategy. New York: Pergamon.

Torabi, S. y Hassini, E. (2008). An interactive possibilistic programming approach for multiple objective supply chain master planning. Fuzzy Sets and Systems, 159(2), 193-214.

Vázquez Núñez, S., \& Vázquez López, S. (2007). Principales enfoques teóricos e investigaciones empíricas generales sobre la internacionalización de PYMES: Un estudio exploratorio de investigaciones entre 1999 y 2004. Contaduría y Administración, 52(222), 41-57.

Veltri, S., Mastroleo, G. y Schaffhauser-Linzatti, M. (2014). Measuring intellectual capital in the university sector using a fuzzy logic expert system. Knowledge Management Research \& Practice, 12(2), 175-192.

Vergara, V., Gaviria, H. (2009). Aplicaciones de la lógica difusa en la planificación de la producción. [trabajo de grado]. Universidad Nacional de Colombia: Medellín.

Wang, R. y Liang, T. (2004). Application of fuzzy multi-objective linear programming to aggregate production planning. Computers \& Industrial Engineering, 46(1), 17-41. 
Wanga, F. y Chang, K. (2010). Adaptive neuro-fuzzy inference system for combined forecasts in a panel manufacturer. Expert Systems with Applications, 37, 8119-8126.

Wernerfelt, B. (1984). A resource-based view of the firm. Strategic Management Journal, 5(2), 171-180.

Wi, H. y Jung, M. (2014). An evaluation fuzzy model and its application for knowledge-based social network. In Social networks: A framework of computational intelligence. Switzerland: Springer International Publishing.

Zadeh, L. (1965). Fuzzy sets. Information and Control, 8, 338-353.

Zadeh, L. (2008). Is there a need for fuzzy logic? Information Sciences, 178(13), 2751-2779. 